\title{
Bases d'un modèle mécaniste des flux d'échanges entre les compartiments ammoniac et urée chez le ruminant
}

\author{
D Sauvant \\ INRA, station de Nutrition et Alimentation, Institut national agronomique (Paris-Grignon), 16, rue \\ Claude-Bernard, 75231 Paris Cedex 05, France
}

\begin{abstract}
Summary - Bases of a mechanistic model of exchange flow between the ammonia and urea compartments in the ruminant. $\mathrm{A}$ mechanistic model of $\mathrm{NH}_{3}$ and urea metabolism in the ruminant has been developed and validated through various literature data bases.
\end{abstract}

La nécessité de mieux intégrer les phénomènes de recyclage ou d'excrétion fécale et urinaire d' $\mathrm{N}$ incite à mettre au point un ou des modèles simples, capables de traduire les principaux flux métaboliques d'NH3 et d'urée chez le ruminant.

Matériel et méthodes - Le modèle comprend 2 compartiments ruminaux (NHR, URR) et 2 métaboliques (NHM, URM) de $\mathrm{NH}_{3}$ et d'urée. Les flux d'entrée dans URR proviennent de l'urée alimentaire ou recyclée par la salive ou la paroi ruminale, ceux entrant dans NHR sont issus du compartiment URR, par uréolyse ruminale, et de la fermentation des fractions azotées alimentaires. Les processus digestifs peuvent être pris en compte de façon statique, à l'aide des relations du système PDI, ou dynamique, en tenant compte des cinétiques d'entrée et de dégradation des substrats, ainsi que leur temps de séjour dans le réticulo-rumen. Le flux d'absorption de $\mathrm{NH}_{3}$ à partir de $\mathrm{NHR}$, à un taux de $90 \% \mathrm{~h}^{-1}$, débouche dans le compartiment NHM, qui alimente directement le compartiment URM. Ce dernier est également approvisionné par la désamination endogène, qui est quantifiée par le bilan PDI de l'animal. L'excrétion d'urée dans le lait à partir d'URM suppose que sa teneur est la même dans le sang et le lait. Le taux de filtration rénale de l'urée à partir d'URM est de $21 \%$ $\mathrm{h}^{-1}$ et sa réabsorption sanguine suit la loi empirique obtenue sur mouton par Scott et Mason (1970). L'urée recyclée par la salive suppose que sa teneur est la même dans le sang et la salive et que $15 \mathrm{I}$ de salive sont produits par $\mathrm{kg}$ de MS ingérée. L'urée recyclée à travers la paroi ruminale dépend, selon une loi michaelienne, de l'urémie. Le taux correspondant est égal à $4,5 \% \mathrm{~h}^{-1}$ lorsque l'urémie est égale à
$80 \mathrm{mg} \mathrm{N} / \mathrm{l}$, valeur de la constante de Michaelis. Le flux d'urée recyclée à travers la paroi des intestins est supposé être égal à $85 \%$ de celui qui traverse la paroi de la panse; en fait, $80 \%$ de ce flux sont directement réabsorbés et se déversent dans le compartiment NHM. Les valeurs des paramètres de base du modèle sont issues de la littérature, les simulations sont effectuées par calculs itératifs de pas de temps d' $1 \mathrm{~min}$.

Résultats et discussion - Le modèle a été validé à travers différents ensembles de données publiées sur les teneurs en urée du plasma, les flux d'urée entrants et recyclés, l'excrétion azotée urinaire... Les résultats sont globalement satisfaisants, à titre illustratif la figure 1 traduit l'aptitude du modèle à prévoir la proportion d'urée recyclée dans le tube digestif et la figure 2 la relation associant le niveau d'excrétion azotée urinaire et l'urémie. L'aspect qui nécessiterait des efforts de recherches concerne, d'après les résultats des simulations, les facteurs de partition des flux d'urée entre les reins et le tube digestif, ainsi qu'entre ses différents segments.

Bertilsson J, Lindberg JE, Gonda H (1991) Proc 6th Int Symp Protein Metabolism and Nutrition. Herning, Danemark, $309 p$

Brun-Bellut J (1986) Thèse Inst Nat Polytechnique de Lorraine

Godwin IR, Williams VJ (1984) $Q J$ Exp Phys 69, 49-59

Harmeyer J, Martens H (1980) J Dairy Sci 63, 1707-1728

Ide Y (1975) Jpn J Vet Sci 37, 327-333 
Lindberg JE, Murphy M (1991) Proc 6th Int Symp Protein Metabolism and Nutrition. Herning, Danemark, $336 \mathrm{p}$

Mc Intyre KH (1970) Aust J Agric Res 21, 501-507

\& du flux recyclé

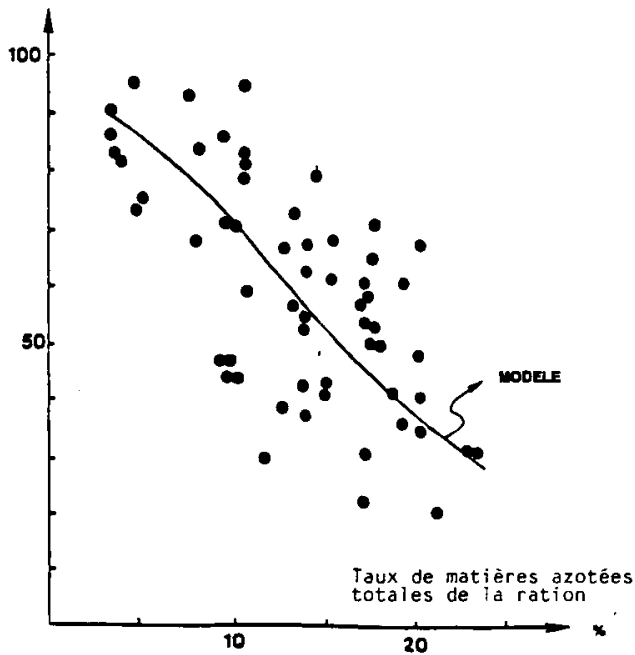

Fig 1. Prévision de la proportion du flux d'urée entrant recyclée dans le tube digestif.

Les valeurs expérimentales mesurées sur bovins, caprins et ovins ont été rassemblées par Harmeyer et Martens (1980).
Rémond B, Journet M (1978) Ann Zootech 27, 139-158

Scott D, Mason GD (1970) Quart J Exp Physiol $55,275-283$

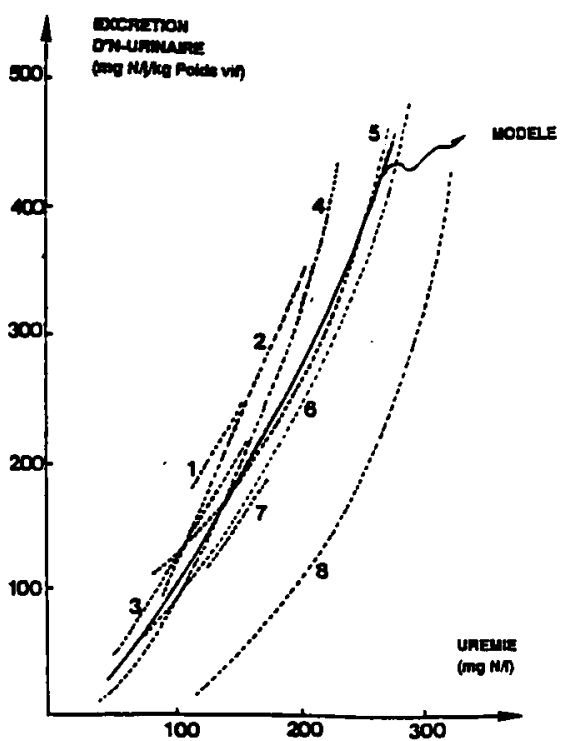

Fig 2. Prévision de l'excrétion azotée urinaire en fonction de l'urémie.

Origine des données : 1) vaches (Bertilsson et al, 1991); 2) vaches (Lindberg et Murphy, 1991); 3) vaches (Rémond, Journet, 1978); 4) chèvres (Ide, 1975); 5) chèvres (Bas et al, 1992, NP); 6) moutons (Mc Intyre, 1970); 7) chèvres (BrunBellut, 1988); 8) moutons (Godwin et Williams, 1984). 